\title{
The First Azacyclopentenylcarbinyl Radical Isomerizations (ACCRI): Independent Use of Steric and Electronic (Polarization) Effects as Gating Elements
}

\author{
Rajesh Viswanathan, Daniel Mutnick, and Jeffrey N. Johnston* \\ Department of Chemistry, Indiana University, Bloomington, IN 47405-7102
}

\section{General Experimental Details}

Flame-dried (under vacuum) glassware was used for all non-aqueous reactions. All reagents and solvents were commercial grade and purified prior to use when necessary. Diethyl ether $\left(\mathrm{Et}_{2} \mathrm{O}\right)$, tetrahydrofuran $(\mathrm{THF})$, dichloromethane $\left(\mathrm{CH}_{2} \mathrm{Cl}_{2}\right)$, and benzene $\left(\mathrm{C}_{6} \mathrm{H}_{6}\right)$ were dried by passage through a column of activated alumina as described by Grubbs. ${ }^{1}$ Benzene was additionally passed through a column containing activated Q-5 reactant. Solvents other than benzene were degassed using the freeze-pump-thaw method when necessary. All additional solvents were dried by distillation from calcium hydride when necessary. Molecular sieves (spheres, $4 \AA$ ) were calcined at $400{ }^{\circ} \mathrm{C}$ and stored at room temperature in an air-tight container. AIBN was recrystallized prior to use, and tri- $n$-butyl tin hydride $\left({ }^{n} \mathrm{Bu}_{3} \mathrm{SnH}\right)$ was used as received from Aldrich. ${ }^{2}$ Magnesium sulfate was used to dry organic solutions unless otherwise noted. Analytical data for $5 \mathbf{a}, 5 \mathbf{b}, \mathbf{5 g}, \mathbf{5 h}, \mathbf{6 a}, \mathbf{6 b}, \mathbf{6 g}, \mathbf{6 h}$ has been previously reported. ${ }^{3}$

Thin layer chromatography (TLC) was performed using glass-backed silica gel $(250 \mu)$ plates, and flash chromatography utilized 230-400 mesh silica gel from Scientific Adsorbents. Neutral Alumina was used as received from Scientific Adsorbents for chromatography of acidsensitive intermediates or products. Products were visualized by UV light, iodine, and/or the use of ceric ammonium molybdate, potassium permanganate, ninhydrin, $p$-anisaldehyde, and potassium iodoplatinate solutions.

\footnotetext{
${ }^{1}$ Pangborn, A. B.; Giardello, M. A.; Grubbs, R. H.; Rosen, R. K.; Timmers, F. J. Organometallics 1996, 15, $1518-1520$.

${ }^{2}$ Two lots (purchased six months apart) from Alfa Aesar failed to effect the radical reactions.

${ }^{3}$ Viswanathan, R.; Plotkin, M. A.; Prabhakaran, E. N.; Johnston, J. N. J. Am. Chem. Soc. 2003, 115, $163-168$.
} 
IR spectra were recorded on a Nicolet Avatar 360 spectrophotometer. Liquids and oils were analyzed as neat films on a salt plate (transmission), whereas solids were applied to a diamond plate (ATR). Nuclear magnetic resonance spectra (NMR) were acquired on either a Varian Inova400 or VXR-400 instrument. Chemical shifts are measured relative to tetramethylsilane, as judged by the residual partially deuterated solvent peak. Mass spectra were obtained using a Kratos MS-80 spectrometer using the ionization technique indicated. Combustion analyses were performed by Atlantic Microlab, Norcross, GA.

Ratios of diastereomers and isomeric products were measured directly from integration of ${ }^{1} \mathrm{H}$ NMR absorptions of protons common to the components. Precision was checked by varying the relaxation delay for measurements on the same compound. Where possible, ratios were corroborated using GC-mass spectrometry. Peak assignments were made from authentic samples in every case. Ratios reported generally represent a lower limit defined by multiple runs. 


\section{General Procedure for Ketimine Condensations}

A rapidly stirred benzene solution of the amine $(0.5 \mathrm{M})$, ketone $(0.5 \mathrm{M})$, and $4 \AA \mathrm{MS}(1: 1$ w/w) was stirred at $25{ }^{\circ} \mathrm{C}$ until complete conversion was achieved, as evidenced by ${ }^{1} \mathrm{H}$ NMR. The mixture was filtered through a pad of Celite and washed with $\mathrm{Et}_{2} \mathrm{O}$ or benzene. The solvent was removed in vacuo to give the analytically pure ketimine which was used immediately.

The same procedure was used when the benzophenone ketimine was desired, except benzophenone imine ${ }^{4}$ was used in place of the ketone. ${ }^{5}$

\section{General Procedure for Asymmetric Phase Transfer Alkylations}

A $0.34 \mathrm{M}$ dichloromethane solution of benzophenone glycinyl imine tert-butyl ester (1 equiv), catalyst ( 0.1 equiv) and solid $\mathrm{CsOH} \cdot \mathrm{H}_{2} \mathrm{O}$ (10 equiv) was cooled to $-78{ }^{\circ} \mathrm{C}$ followed by dropwise addition of benzyl halide ( 5 equiv). The heterogeneous mixture was stirred for the time specified while maintaining the low temperature. The solution was diluted with $\mathrm{Et}_{2} \mathrm{O}$, washed with water, dried and concentrated prior to purification by chromatography (neutral alumina) which gave analytically pure ketimine. Absolute stereochemistry was assigned be analogy.

\section{General Procedure for Cyclizations Using Chiral Non-racemic Schiff Base}

\section{Substrates}

A benzene solution of the ketimine ( 1 equiv) and ${ }^{n} \mathrm{Bu}_{3} \mathrm{SnH}$ (2.2 equiv) was warmed to 85 ${ }^{\circ} \mathrm{C}$ followed by slow addition of AIBN (1.2 equiv) using a syringe pump over $4-5 \mathrm{hrs}$. The solution was refluxed for an additional hour before concentration. The residue was treated with a 1:1 (by volume) solution of $\mathrm{Et}_{2} \mathrm{O}$ and satd aq $\mathrm{KF}^{6}$ and the mixture stirred vigorously until a white solid precipitated. The organic layer was washed with water, and concentrated prior to purification by silica gel chromatography to give the target indoline.

\section{General Procedure for Preparation of Racemic Samples}

A $0.34 \mathrm{M}$ dichloromethane solution of benzophenone glycinyl imine tert-butyl ester (1 equiv), benzyltriethyl ammonium chloride ( 0.2 equiv), and benzyl halide (1.2 equiv) was treated

\footnotetext{
${ }^{4}$ Pickard, P. L.; Tolbert, T. L. in "Organic Syntheses”; Wiley: NY, 1973, Collective Vol. 5, pp. 520-2.

5 O’Donnell, M. J.; Polt, R. L. J. Org. Chem. 1982, 47, 2663.

${ }^{6}$ Complete saturation by KF is necessary.
} 
with $50 \%$ aq $\mathrm{NaOH}$ (20 equiv). The mixture was stirred vigorously at room temperature for $4 \mathrm{~h}$. The heterogeneous mixture was diluted with $\mathrm{Et}_{2} \mathrm{O}$, washed with water, dried, and concentrated prior to purification by column chromatography (neutral alumina) to yield pure racemic ketimines.

General Procedure for Imine Reduction (when necessary to determine ee)

To a $0.02 \mathrm{M}$ solution of ketimine ( 1 equiv) in dry methanol, was added $\mathrm{NaBH}_{4}$ (25 equiv) at $0{ }^{\circ} \mathrm{C}$. The mixture was warmed up to $25^{\circ} \mathrm{C}$, and stirred for $1-1.5 \mathrm{~h}$. The solution was diluted with $\mathrm{CH}_{2} \mathrm{Cl}_{2}$, washed with water, dried, and concentrated prior to purification by silica gel chromatography to yield analytically pure amine for HPLC analysis. 


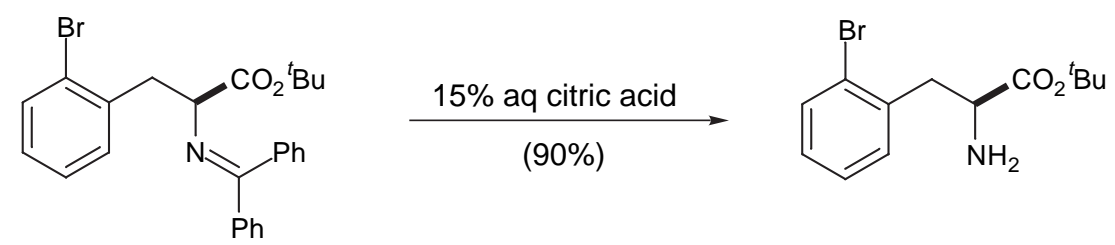

(S)-2-Amino-3-(2-bromophenyl)-propionic acid tert-butyl ester (S-1). ${ }^{7}$ Imine (S)5a $(599 \mathrm{mg}, 1.29 \mathrm{mmol})$ was dissolved in THF $(7.7 \mathrm{~mL})$ and treated with aq 15\% citric acid (3.9 $\mathrm{mL})$. The mixture was stirred vigorously for $5 \mathrm{~h}$ at room temperature, diluted with water $(10 \mathrm{~mL})$ and extracted twice with ether. The $\mathrm{pH}$ of the aqueous layer was adjusted from 2 to 10 by dropwise addition of satd $\mathrm{K}_{2} \mathrm{CO}_{3}$ solution. The aqueous layer was thoroughly extracted with ethyl acetate, the combined organic layers were dried and concentrated, and the residue was purified by silica gel chromatography ( $2 \%$ methanol in dichloromethane) to furnish the amine as a colorless oil (348 mg, 90\%). $\mathrm{R}_{f}=0.67$ (10\% MeOH/hexanes); IR (film) 3382, $1733 \mathrm{~cm}^{-1} ;{ }^{1} \mathrm{H}$ NMR (400 MHz, $\left.\mathrm{CDCl}_{3}\right) \delta 7.54(\mathrm{~d}, J=8.3 \mathrm{~Hz}, 1 \mathrm{H}), 7.26-7.21(\mathrm{~m}, 2 \mathrm{H}), 7.11-7.07(\mathrm{~m}, 1 \mathrm{H}), 3.73(\mathrm{t}, J=7.3 \mathrm{~Hz}$, $1 \mathrm{H}), 3.20(\mathrm{dd}, J=13.6,5.8 \mathrm{~Hz}, 1 \mathrm{H}), 2.87(\mathrm{dd}, J=13.6,8.3 \mathrm{~Hz}, 1 \mathrm{H}), 1.53(\mathrm{br}, \mathrm{s}, 2 \mathrm{H}) 1.41(\mathrm{~s}$, 9H); ${ }^{13} \mathrm{C}$ NMR (100 MHz, $\mathrm{CDCl}_{3}$ ) ppm 174.1, 137.4, 132.8, 131.6, 128.2, 127.2, 124.8, 81.1, 54.8, 41.7, 27.9; HRMS (EI): Exact mass calcd for $\mathrm{C}_{13} \mathrm{H}_{19} \mathrm{BrNO}_{2}[\mathrm{M}+\mathrm{H}]^{+}$, 300.0599. Found 300.0647. HPLC (Chiralcel AD, 5\% 'PrOH/hexanes, $1 \mathrm{mLmin}): \mathrm{t}_{\mathrm{r}}(R)=9.2 \mathrm{~m}, \mathrm{t}_{\mathrm{r}}(S)=10.3 \mathrm{~m}$. $95 \%$ ee: $[\alpha]_{\mathrm{D}}^{21}+29.3^{\circ}\left(c 1.0, \mathrm{CHCl}_{3}\right)$.

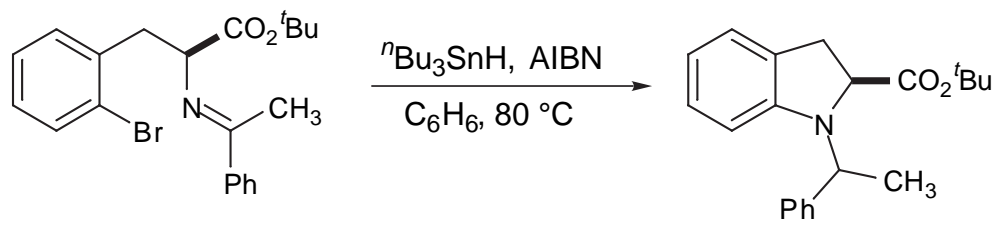

1-(1-phenylethyl)-2,3-dihydro-1H-indole-2-carboxylic acid tert-butyl ester (6d). According to the general procedure, use of $\mathbf{5 d}(42 \mathrm{mg}, 104 \mu \mathrm{mol}),{ }^{\mathrm{n}} \mathrm{Bu}_{3} \mathrm{SnH}(62 \mu \mathrm{L}, 229 \mu \mathrm{mol})$, and AIBN $(13.7 \mathrm{mg}, 83.3 \mu \mathrm{mol})$ provided the desired indoline after flash chromatography $\left(\mathrm{SiO}_{2}\right.$, $1 \%$ ethyl acetate in hexanes) as a yellow oil $(\mathrm{dr}=5: 1,25.7 \mathrm{mg}, 76 \%) . \mathrm{R}_{f}=0.33(10 \%$ ethyl actetate in hexanes); IR (film) 2977, 2928, 1742, $1606 \mathrm{~cm}^{-1} ;{ }^{1} \mathrm{H}$ NMR (400 MHz, $\mathrm{CDCl}_{3}$ ) $\delta$ (major

\footnotetext{
${ }^{7}$ Lygo, B.; Wainwright, P.G. Tetrahedron. Lett. 1997, 38, 8595.
} 
diastereomer) $7.54(\mathrm{~d}, J=7.5 \mathrm{~Hz}, 2 \mathrm{H}), 7.34(\mathrm{dd}, J=7.8,7.4 \mathrm{~Hz}, 2 \mathrm{H}), 7.24(\mathrm{~m}, 1 \mathrm{H}), 7.02(\mathrm{~d}, J$ $=7.3 \mathrm{~Hz}, 1 \mathrm{H}), 6.93(\mathrm{t}, J=7.7 \mathrm{~Hz}, 1 \mathrm{H}), 6.64(\mathrm{dd}, J=7.4,7.3 \mathrm{~Hz}, 1 \mathrm{H}), 6.21(\mathrm{~d}, J=7.9 \mathrm{~Hz}$, $1 \mathrm{H}), 4.50(\mathrm{q}, J=7.1 \mathrm{~Hz}, 1 \mathrm{H}), 4.40(\mathrm{dd}, J=9.8,9.6 \mathrm{~Hz}, 1 \mathrm{H}), 3.43(\mathrm{dd}, J=15.7,10.3 \mathrm{~Hz}$, 1H), $3.09(\mathrm{dd}, J=15.7,9.3 \mathrm{~Hz}, 1 \mathrm{H}), 1.54(\mathrm{~d}, J=7.0 \mathrm{~Hz}, 3 \mathrm{H}), 1.40(\mathrm{~s}, 9 \mathrm{H}) ;{ }^{13} \mathrm{C}$ NMR $(100$ MHz) ppm 173.7, 151.9, 144.5, 128.7, 127.8, 127.5, 127.3, 127.1, 124.0, 118.2, 108.8, 81.3, 64.6, 58.7, 34.7, 29.9, 28.6, 28.1, 19.8; HRMS (CI, $\mathrm{CH}_{4}$ ) Exact mass calcd for $\mathrm{C}_{21} \mathrm{H}_{25} \mathrm{NO}_{2}$ $[\mathrm{M}]^{+}$323.1885. Found 323.1895.

Anal. Calcd. for $\mathrm{C}_{21} \mathrm{H}_{25} \mathrm{~N}$ : C, 77.98; H, 7.79; N, 4.33. Found C, 78.03; H, 7.90; N, 4.44 .

HPLC (Chiralcel AD, 5\% ${ }^{~} \mathrm{PrOH} /$ hexanes, $\left.1 \mathrm{~mL} / \mathrm{min}\right)$ : Diastereomer 1: $\mathrm{t}_{\mathrm{r}}(S)=3.89 \mathrm{~m}, \mathrm{t}_{\mathrm{r}}$ $(R)=4.03 \mathrm{~m}$; Diastereomer 2: $\mathrm{t}_{\mathrm{r}}(S)=4.65 \mathrm{~m}, \mathrm{t}_{\mathrm{r}}(R)=4.92 \mathrm{~m} ; 92 \%$ ee: $[\alpha]_{\mathrm{D}}^{24}-2.2^{\circ}(c 0.5$, $\left.\mathrm{CHCl}_{3}\right)$.
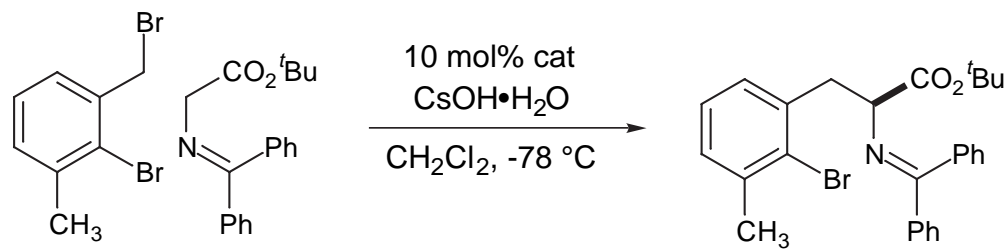

(S)-2-(Benzhydrylideneamino)-1-(2-bromo-3-methylphenyl)propionic acid tertbutyl ester (5c). Following the general procedure, 2-bromo-3-bromomethyltoluene ${ }^{8}$ (750 $\mathrm{mg}$, $2.84 \mathrm{mmol})$, glycinyl imine (168 mg, $0.568 \mathrm{mmol})$, and $\mathrm{CsOH} \cdot \mathrm{H}_{2} \mathrm{O}(847 \mathrm{mg}, 5.68 \mathrm{mmol})$ were stirred in $\mathrm{CH}_{2} \mathrm{Cl}_{2}$ for $20 \mathrm{~h}$ in the presence of the cinchonidine derived catalyst $(10 \mathrm{~mol} \%)$ while maintaining a temperature $\leq-78{ }^{\circ} \mathrm{C}$. Work-up according to the general procedure provided the desired product as a white solid (233 mg, 86\%). $\mathrm{mp}=92-94{ }^{\circ} \mathrm{C} ; \mathrm{R}_{f}=0.38(10 \% \mathrm{EtOAc} / \mathrm{hexanes})$; IR (film) 3056, $1732 \mathrm{~cm}^{-1} ;{ }^{1} \mathrm{H} \mathrm{NMR}\left(400 \mathrm{MHz}, \mathrm{CDCl}_{3}\right) 7.59$ (dd, $\left.J=7.9,1.5 \mathrm{~Hz}, 2 \mathrm{H}\right)$, 7.40-7.25 (m, 6H), 7.09-7.04 (m, 3H), 6.55 (br d, J=5.0 Hz, 2H), $4.42(\mathrm{dd}, J=9.8,3.9 \mathrm{~Hz}$, $1 \mathrm{H}), 3.53(\mathrm{dd}, J=13.3,3.9 \mathrm{~Hz}, 1 \mathrm{H}), 3.24(\mathrm{dd}, J=13.4,9.9 \mathrm{~Hz}, 1 \mathrm{H}), 2.35$ (s, 3H), 1.49 (s,

\footnotetext{
${ }^{8}$ 2-Bromo- $m$-xylene was converted to the corresponding benzyl bromide: Rebek, J.; Costello, T.; Wattley, R. $J$. Am. Chem. Soc. 107, 1985, 7487.
} 
9H); ${ }^{13} \mathrm{C}$ NMR (100 MHz, $\left.\mathrm{CDCl}_{3}\right)$ 170.8, 170.5, 139.5, 138.2, 137.8, 136.2, 130.2, 130.0, 128.8, 128.7 (2C), 128.1, 127.9 (2C), 127.6 (2C), 126.3, 81.1, 64.9, 40.2, 28.1, 23.9; HRMS (EI): Exact mass calcd for $\mathrm{C}_{27} \mathrm{H}_{29} \mathrm{BrNO}_{2}[\mathrm{M}+\mathrm{H}]^{+}$, 478.1382. Found 478.1325.

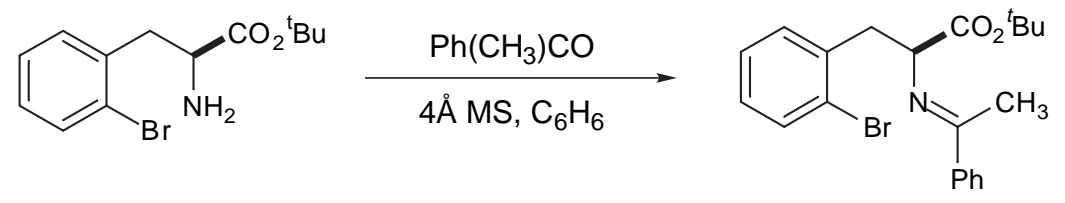

3-(2-bromo-phenyl)-2-(1-phenyl-ethylideneamino)-propionic acid tert-butyl ester (5d). According to the general procedure, use of $\mathbf{S - 1}(179 \mathrm{mg}, 600 \mu \mathrm{mol})$, acetophenone (77 $\mu \mathrm{L}$, $660 \mu \mathrm{mol})$ and $4 \AA \mathrm{MS}(1.5 \mathrm{~g})$, in benzene $(5 \mathrm{ml})$ gave the desired imine as a yellow oil (221 $\mathrm{mg}$, 92\%). IR (film) 2977, 1731, $1150 \mathrm{~cm}^{-1} ;{ }^{1} \mathrm{H}$ NMR (400 MHz, $\mathrm{CDCl}_{3}$ ) $\delta 7.72$ (ddd, $J=3.4,3.4$, $1.5 \mathrm{~Hz}, 2 \mathrm{H}), 7.53(\mathrm{dd}, J=7.9,1.2 \mathrm{~Hz}, 1 \mathrm{H}), 7.36(\mathrm{~m}, 2 \mathrm{H}), 7.24(\mathrm{~m}, 2 \mathrm{H}), 7.16(\mathrm{~m}, 1 \mathrm{H}), 7.07$ $(\mathrm{m}, 1 \mathrm{H}), 4.63(\mathrm{dd}, J=8.5,5.2 \mathrm{~Hz}, 1 \mathrm{H}), 3.59(\mathrm{dd}, J=13.4,5.5 \mathrm{~Hz}, 1 \mathrm{H}), 3.18(\mathrm{dd}, J=13.4$, $8.8 \mathrm{~Hz}, 1 \mathrm{H}), 1.96$ (s, 3H), 1.45 (s, 9H); ${ }^{13} \mathrm{C} \mathrm{NMR}\left(100 \mathrm{MHz}, \mathrm{CDCl}_{3}\right.$ ) ppm 173.7, 151.9, 144.5, $128.7,127.8,127.5,127.3,127.1,124.0,118.2,108.8,81.3,64.6,58.7,34.7,29.9,28.6$, 28.1, 19.8; HRMS (EI): Exact mass calcd for $\mathrm{C}_{21} \mathrm{H}_{25} \mathrm{BrNO}_{2}[\mathrm{M}+\mathrm{H}]^{+}$402.1070. Found 402.1071.
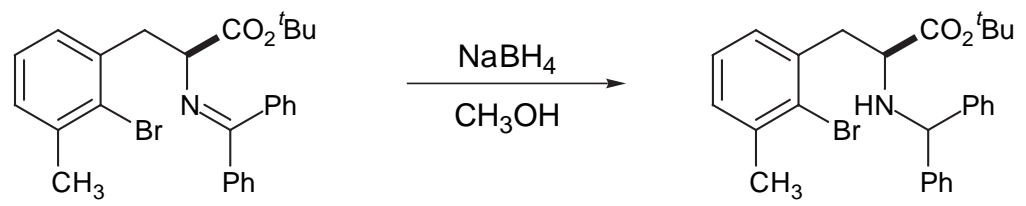

(S)-2-(Benzhydrylamino)-3-(2-bromo-3-methylphenyl)propionic acid tert-butyl ester (S-2). The enantiomeric ratio was determined after reduction of the ketimine. Addition of $\mathrm{NaBH}_{4}(3.77 \mathrm{mg}, 99.5 \mu \mathrm{mol})$ to imine $(9.50 \mathrm{mg}, 19.9 \mu \mathrm{mol})$ in $\mathrm{MeOH}(1.0 \mathrm{~mL})$ furnished, after flash chromatography $\left(\mathrm{SiO}_{2}, 2 \%\right.$ ethyl acetate in hexanes), the amine as a white solid ( $\left.8 \mathrm{mg}, 83 \%\right)$. mp 116-118 ${ }^{\circ} \mathrm{C} ; \mathrm{R}_{f}=0.43$ (10\% EtOAc/hexanes); IR (film) 3323, $1723 \mathrm{~cm}^{-1} ;{ }^{1} \mathrm{H}$ NMR (400 MHz, $\left.\mathrm{CDCl}_{3}\right) \delta 7.34(\mathrm{dd}, J=8.2,1.5 \mathrm{~Hz}, 2 \mathrm{H}), 7.28(\mathrm{td}, J=6.3,1.8 \mathrm{~Hz}, 2 \mathrm{H}), 7.22(\mathrm{t}, J=1.3 \mathrm{~Hz}$, 1H), 7.20-7.13, (m, 7H), 7.09-7.07 (m, 1H), $4.81(\mathrm{~s}, 1 \mathrm{H}), 3.50(\mathrm{t}, J=7.7 \mathrm{~Hz}, 1 \mathrm{H}), 3.13$ (dd, 
$J=13.4,6.9 \mathrm{~Hz}, 1 \mathrm{H}), 3.06(\mathrm{dd}, J=13.4,5.0 \mathrm{~Hz}, 1 \mathrm{H}), 2.43(\mathrm{~s}, 3 \mathrm{H}), 2.21(\mathrm{br} \mathrm{s}, 1 \mathrm{H}), 1.42(\mathrm{~s}$, 9H); ${ }^{13} \mathrm{C}$ NMR (100 MHz, $\mathrm{CDCl}_{3}$ ) ppm 174.1, 138.4, 138.0, 129.6, 128.9, 128.4, 128.2, $127.7,127.4,127.3,127.1,126.3,117.9,112.8,95.4,81.1,65.4,59.4,41.0,28.0,24.0$; HRMS (EI): Exact mass calcd for $\mathrm{C}_{27} \mathrm{H}_{31} \mathrm{BrNO}_{2}[\mathrm{M}+\mathrm{H}]^{+}$480.1538. Found 480.1560.

HPLC (Chiralcel OD, 2\% ${ }^{i} \mathrm{PrOH} /$ hexanes, $\left.1 \mathrm{mLmin}\right): \mathrm{t}_{\mathrm{r}}(S)=4.9 \mathrm{~m}, \mathrm{t}_{\mathrm{r}}(R) 5.6 \mathrm{~m}$. $(S)-88 \%$ ee $[\alpha]_{\mathrm{D}}^{23}-35.2\left(c 0.8, \mathrm{CHCl}_{3}\right) .(R)-86 \%$ ee, $[\alpha]_{\mathrm{D}}^{24}+33.2\left(c 0.5, \mathrm{CHCl}_{3}\right)$.
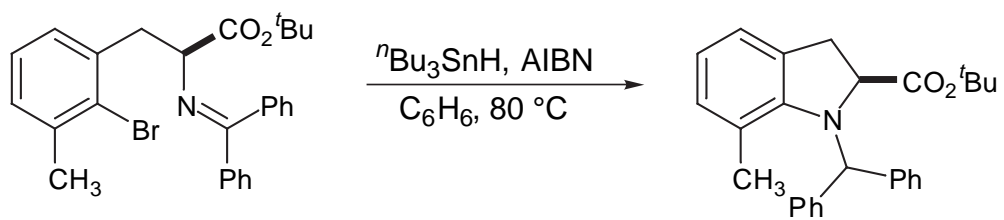

\section{1-Benzhydryl-7-methyl-2,3-dihydro-1H-indole-2-carboxylic acid tert-butyl ester}

(6c). Following the general procedure, use of the ketimine $(41.0 \mathrm{mg}, 86.0 \mu \mathrm{mol}),{ }^{n} \mathrm{Bu}_{3} \mathrm{SnH}(51.0$ $\mu \mathrm{L}, 0.189 \mathrm{mmol}$ ), and AIBN (17 mg, $0.103 \mathrm{mmol})$ provided, following chromatography $\left(\mathrm{SiO}_{2}\right.$, $1 \%$ ethyl acetate in hexanes), the racemic indoline as a colorless oil $(5.00 \mathrm{mg}, 15 \%) .{ }^{9} \mathrm{R}_{f}=0.40$ (10\% EtOAc/hexanes); IR (film) 3059, $1743 \mathrm{~cm}^{-1} ;{ }^{1} \mathrm{H}$ NMR (400 MHz, $\left.\mathrm{CDCl}_{3}\right) \delta 7.58$ (d, J= 7.9 $\mathrm{Hz}, 2 \mathrm{H}), 7.39-7.30(\mathrm{~m}, 4 \mathrm{H}), 7.26-7.20(\mathrm{~m}, 2 \mathrm{H}), 6.97(\mathrm{td}, J=7.8,1.1 \mathrm{~Hz}, 3 \mathrm{H}), 6.85(\mathrm{~d}, J=$ $7.1 \mathrm{~Hz}, 1 \mathrm{H}), 6.79(\mathrm{t}, J=7.4 \mathrm{~Hz}, 1 \mathrm{H}), 5.95(\mathrm{~s}, 1 \mathrm{H}), 4.05(\mathrm{dd}, J=7.3,5.2 \mathrm{~Hz}, 1 \mathrm{H}), 2.61(\mathrm{~d}, J=$ $5.9 \mathrm{~Hz}, 2 \mathrm{H}), 2.37$ (s, 3H), 1.39 (s, 9H); ${ }^{13} \mathrm{C} \mathrm{NMR}\left(100 \mathrm{MHz}, \mathrm{CDCl}_{3}\right.$ ) ppm 173.2, 150.9, 141.2, $140.4,132.1,130.1,128.9,128.7,128.2,128.0,127.4,126.9,123.6,121.6,121.5,80.5$, 68.2, 61.7, 35.0, 27.9, 18.5; HRMS (EI): Exact mass calcd for $\mathrm{C}_{27} \mathrm{H}_{29} \mathrm{NO}_{2}[\mathrm{M}]^{+}, 399.2198$. Found 399.2203.

HPLC (Chiralcel AD, 0.5\% ${ }^{~} \mathrm{PrOH} /$ hexanes, $\left.0.5 \mathrm{mLmin}\right): \mathrm{t}_{\mathrm{r}}(S)=10.0 \mathrm{~m}, \mathrm{t}_{\mathrm{r}}(R)=10.6 \mathrm{~m}$.

\footnotetext{
${ }^{9}$ The mass balance consisted of un-reacted starting material and reduced aryl bomide (ArH).
} 


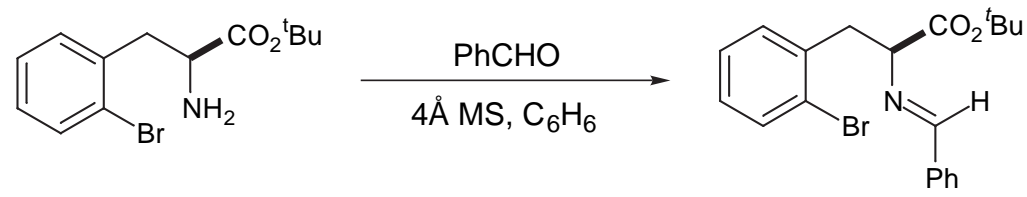

2-(Benzylidene amino)-3-(2-bromophenyl)-propionic acid tert-butyl ester (5e). Following the general procedure (Method A), amine S-1 (250 mg, $833 \mu \mathrm{mol})$, benzaldehyde $(85.0 \mu \mathrm{L}, 833 \mu \mathrm{mol})$, and $4 \AA \mathrm{MS}$ in benzene $(0.8 \mathrm{~mL})$ after $10 \mathrm{~h}$ provided the aldimine $(330 \mathrm{mg}$, 100\%), as a >95: 5 mixture of stereoisomers and a colorless oil. IR (film) 3062, 1733, $1641 \mathrm{~cm}^{-1}$; ${ }^{1} \mathrm{H}$ NMR (400 MHz, $\left.\mathrm{CDCl}_{3}\right) \delta 8.00(\mathrm{~s}, 1 \mathrm{H}), 7.71(\mathrm{dd}, J=7.7,1.5 \mathrm{~Hz}, 2 \mathrm{H}), 7.53(\mathrm{~d}, J=7.8 \mathrm{~Hz}$, $1 \mathrm{H}), 7.42-7.35(\mathrm{~m}, 3 \mathrm{H}) ; 7.21(\mathrm{~d}, J=7.5 \mathrm{~Hz}, 1 \mathrm{H}), 7.14(\mathrm{t}, J=7.4 \mathrm{~Hz}, 1 \mathrm{H}), 7.04(\mathrm{td}, J=8.9$, $1.3 \mathrm{~Hz}, 1 \mathrm{H}), 4.25(\mathrm{dd}, J=8.2,5.8 \mathrm{~Hz}, 1 \mathrm{H}), 3.56(\mathrm{dd}, J=13.6,5.8 \mathrm{~Hz}, 1 \mathrm{H}), 3.19(\mathrm{dd}, J=$ 13.6, $8.3 \mathrm{~Hz}, 1 \mathrm{H}) 1.45$ (s, 9H); ${ }^{13} \mathrm{C}$ NMR (100 MHz, $\mathrm{CDCl}_{3}$ ) ppm 170.5, 163.5, 137.1, 135.7, 132.6, 130.9, 128.4, 128.3, 128.2, 127.0, 124.8, 81.4, 72.9, 39.8, 28.0; HRMS (EI): Exact mass calcd for $\mathrm{C}_{20} \mathrm{H}_{23} \mathrm{BrNO}_{2}[\mathrm{M}+\mathrm{H}]^{+}$, 388.0912. Found 388.0917.

HPLC (Chiralcel OD, 2\% ${ }^{i} \mathrm{PrOH} /$ hexanes, $\left.1 \mathrm{mLmin}\right): \mathrm{t}_{\mathrm{r}}(S)=5.4 \mathrm{~m}, \mathrm{t}_{\mathrm{r}}(R)=6.3 \mathrm{~m} .(S)-$ $94 \%$ ee $[\alpha]_{\mathrm{D}}^{21}-140.1^{\circ}\left(c 1.5, \mathrm{CHCl}_{3}\right)$.

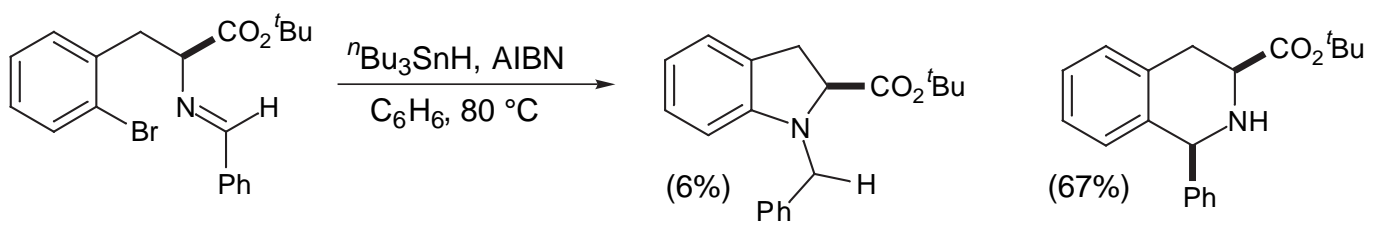

Following the general procedure, use of aldimine 5e (224 mg, $577 \mu \mathrm{mol}),{ }^{n} \mathrm{Bu}_{3} \mathrm{SnH}(171 \mu \mathrm{L}, 635$ $\mu \mathrm{mol}$ ), and AIBN (76.0 mg, $462 \mu \mathrm{mol})$ furnished, after silica gel chromatography (3-5\% ethyl acetate in hexanes, the indoline $\mathbf{6 e}(10.4 \mathrm{mg}, 6 \%)$ as a colorless oil and tetrahydroisoquinoline $\mathbf{S}$ $3(119.0 \mathrm{mg}, 67 \%)$ as a colorless solid.

(S)-1-Benzyl-2,3-dihydro-1-H-indole-2-carboxylic acid tert-butyl ester (6e). $\mathrm{R}_{f}=0.42$ (10\% EtOAc/hexanes); IR (film) 3026, $1736 \mathrm{~cm}^{-1} ;{ }^{1} \mathrm{H}$ NMR (400 MHz, $\left.\mathrm{CDCl}_{3}\right) \delta 7.38$ $(\mathrm{d}, J=7.0 \mathrm{~Hz}, 1 \mathrm{H}), 7.33(\mathrm{t}, J=7.0 \mathrm{~Hz}, 2 \mathrm{H}), 7.28-7.24(\mathrm{~m}, 2 \mathrm{H}), 7.07-7.01(\mathrm{~m}, 2 \mathrm{H}), 6.68(\mathrm{t}, J$ $=7.5 \mathrm{~Hz}, 1 \mathrm{H}), 6.40(\mathrm{~d}, J=7.8 \mathrm{~Hz}, 1 \mathrm{H}), 4.56(\mathrm{~d}, J=15.6 \mathrm{~Hz}, 1 \mathrm{H}), 4.30(\mathrm{~d}, J=15.7 \mathrm{~Hz}, 1 \mathrm{H})$ 
$4.16(\mathrm{dd} J=10.2,8.2 \mathrm{~Hz}, 1 \mathrm{H}), 3.36(\mathrm{dd}, J=15.9,10.2 \mathrm{~Hz}, 1 \mathrm{H}), 3.19(\mathrm{dd}, J=15.9,8.2 \mathrm{~Hz}$, $1 \mathrm{H}), 1.43(\mathrm{~s}, 9 \mathrm{H}) ;{ }^{13} \mathrm{C} \mathrm{NMR}\left(100 \mathrm{MHz}, \mathrm{CDCl}_{3}\right) \mathrm{ppm} 171.9,151.6,130.3,128.5,127.71$, $127.67,127.1,124.1,122.4$,

117.9, 107.0, 81.4, 66.2, 52.1, 33.4, 28.0; HRMS (EI): Exact mass calcd for $\mathrm{C}_{20} \mathrm{H}_{23} \mathrm{NO}_{2}[\mathrm{M}]^{+}$, 309.1729. Found 309.1744.

HPLC (Chiralcel OD, $1 \%{ }^{i}$ PrOH/hexanes, $\left.0.5 \mathrm{mLmin}\right): \mathrm{t}_{\mathrm{r}}(S)=14.5 \mathrm{~m}, \mathrm{t}_{\mathrm{r}}(R)=17.7 \mathrm{~m}$. $(S)-81 \%$ ee, $[\alpha]_{\mathrm{D}}^{25}-5.3^{\circ}\left(c 0.3, \mathrm{CHCl}_{3}\right)$.

(S)-1-Phenyl-1,2,3,4-tetrahydro isoquinoline-3-carboxylic acid tert-butyl ester (S-3). mp 116-119 ${ }^{\circ} \mathrm{C} ; \mathrm{R}_{f}=0.25$ (10\% EtOAc/hexanes); IR (film) 2975, $1726 \mathrm{~cm}^{-1} ;{ }^{1} \mathrm{H}$ NMR (400 $\left.\mathrm{MHz}, \mathrm{CDCl}_{3}\right) \delta 7.40-7.30(\mathrm{~m}, 5 \mathrm{H}), 7.16(\mathrm{dd}, J=14.4,7.5 \mathrm{~Hz}, 2 \mathrm{H}), 7.05(\mathrm{td}, J=7.9,1.9 \mathrm{~Hz}$, $1 \mathrm{H}), 6.72(\mathrm{~d}, J=7.8 \mathrm{~Hz}, 1 \mathrm{H}), 5.14(\mathrm{~s}, 1 \mathrm{H}), 3.81(\mathrm{dd}, J=9.0,6.5 \mathrm{~Hz}, 1 \mathrm{H}), 3.15(\mathrm{~d}, J=6.9$ $\mathrm{Hz}, 2 \mathrm{H}), 2.47$ (br, s, 1H), 1.54 (s, 9H); ${ }^{13} \mathrm{C} \mathrm{NMR} \mathrm{(100} \mathrm{MHz,} \mathrm{CDCl}_{3}$ ) ppm 171.7, 143.9, 138.5, $134.1,129.1,128.8,128.4,127.6,127.5,126.2,125.9,81.5,63.2,57.0,32.8,28.0$; HRMS (EI): Exact mass calcd for $\mathrm{C}_{20} \mathrm{H}_{23} \mathrm{NO}_{2}[\mathrm{M}]^{+}, 309.1729$. Found 309.1715.

Anal. Calcd for $\mathrm{C}_{20} \mathrm{H}_{23} \mathrm{NO}_{2}$ : C, 77.64, H, 7.49, N, 4.53. Found : C, 77.36, H, 7.55, N, 4.43 .

HPLC (Chiralcel AD, 10\% ${ }^{i} \operatorname{PrOH} /$ hexanes, $\left.1.0 \mathrm{mLmin}\right): \mathrm{t}_{\mathrm{r}}(S)=6.0 \mathrm{~m}, \mathrm{t}_{\mathrm{r}}(R)=8.9 \mathrm{~m}$. $(S)-95 \%$ ee, $[\alpha]_{\mathrm{D}}^{25}-8.8^{\circ}\left(c 1.0, \mathrm{CHCl}_{3}\right)$.
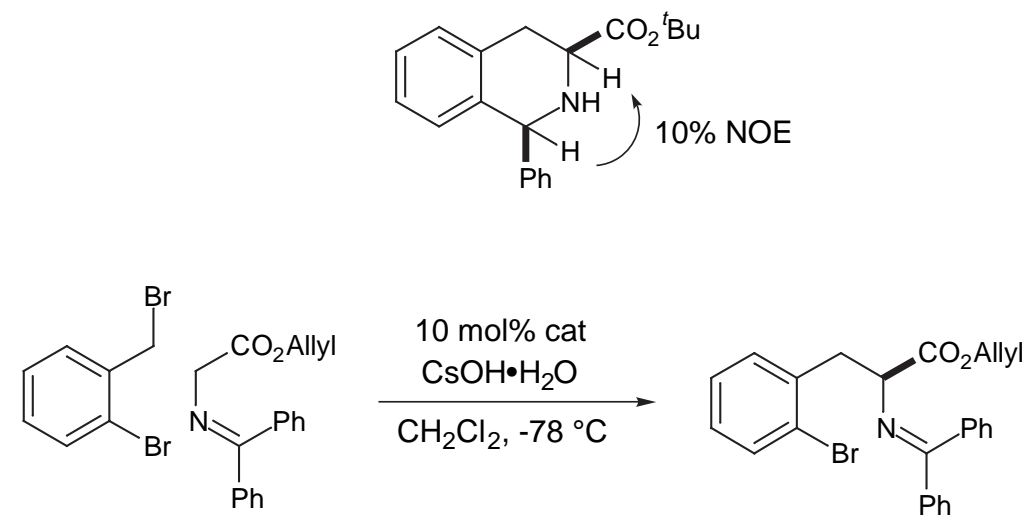

2-(Benzhydrylidene-amino)-3-(2-bromophenyl)-propionic acid allyl ester (5f). Following the general procedure, ortho-bromobenzyl bromide $(501 \mathrm{mg}, 1.79 \mu \mathrm{mol})$, glycine allyl 
ester benzophenone imine (100 mg, $357 \mu \mathrm{mol}), \mathrm{CsOH} \bullet \mathrm{H}_{2} \mathrm{O}(532 \mathrm{mg}, 3.57 \mathrm{mmol}$ ), and the cinchonidine derived catalyst $(21.8 \mathrm{mg}, 36.0 \mathrm{mmol})$ were stirred in $\mathrm{CH}_{2} \mathrm{Cl}_{2}(1.05 \mathrm{~mL})$ at-78 ${ }^{\circ} \mathrm{C}$ for 7 hours. Workup according to the general procedure and flash chromatography (neutral alumina, $4 \%$ ethyl acetate in hexanes) gave the desired phenyl alanine derivative as a colorless oil (100 mg, 62\% yield). $\mathrm{R}_{f}=0.20$ (10\% EtOAc/hexanes); IR (film) 3062, 1748, $1618 \mathrm{~cm}^{-1} ;{ }^{1} \mathrm{H}$ NMR $\left(400 \mathrm{MHz}, \mathrm{CDCl}_{3}\right) \delta 7.64(\mathrm{~d}, J=7.3 \mathrm{~Hz}, 2 \mathrm{H}), 7.45(\mathrm{~d}, J=7.9 \mathrm{~Hz}, 1 \mathrm{H}), 7.39(\mathrm{t}, J=6.3 \mathrm{~Hz}$, 2H), 7.36- $7.30(\mathrm{~m}, 4 \mathrm{H}), 7.23(\mathrm{~d}, J=7.5 \mathrm{~Hz}, 1 \mathrm{H}), 7.16(\mathrm{t}, J=7.3 \mathrm{~Hz}, 1 \mathrm{H}), 7.06(\mathrm{td}, J=8.8$, 1.5, Hz, 1H), 6.62 (bs s, 2H), $5.94(\mathrm{ddd}, J=18.3,10.5,5.6 \mathrm{~Hz}, 1 \mathrm{H}), 5.34(\mathrm{dd}, J=18.3,1.1$ Hz, 1H), 5.25 (d, $J=10.5 \mathrm{~Hz}, 1 \mathrm{H})$; 4.74-4.65 (m, 2H), $4.58(\mathrm{dd}, J=9.8,3.8 \mathrm{~Hz}, 1 \mathrm{H}), 3.58$ $(\mathrm{dd}, J=13.2,4.2 \mathrm{~Hz}, 1 \mathrm{H}), 3.31(\mathrm{dd}, J=13.2,10.0 \mathrm{~Hz}, 1 \mathrm{H}) ;{ }^{13} \mathrm{C} \mathrm{NMR}\left(100 \mathrm{MHz}, \mathrm{CDCl}_{3}\right) \mathrm{ppm}$ $171.2,171.1,139.1,137.0,135.7,132.7,132.5,131.9,130.9,130.0,128.7,128.3,128.2$, 128.1 (2C), 127.5, 126.9, 118.0, 65.5, 64.3, 39.5; HRMS (EI): Exact mass calcd for $\mathrm{C}_{25} \mathrm{H}_{23} \mathrm{BrNO}_{2}[\mathrm{M}+\mathrm{H}]^{+}, 448.0912$. Found 448.0915.

HPLC (Chiralcel OD, $2 \%{ }^{i} \operatorname{PrOH} /$ hexanes, $\left.1 \mathrm{mLmin}\right): \mathrm{t}_{\mathrm{r}}(R)=5.9 \mathrm{~m}, \mathrm{t}_{\mathrm{r}}(S)=6.6 \mathrm{~m} .(S)-$ $76 \%$ ee $[\alpha]_{\mathrm{D}}^{21}-265.5^{\circ}\left(c 0.5, \mathrm{CHCl}_{3}\right)$.

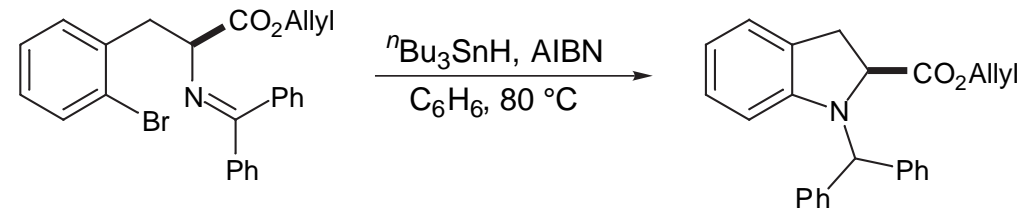

1-Benzhydryl-2,3-dihydro-1H-indole-2-carboxylic acid allyl ester (6f). According to the general procedure, Schiff base $(40.2 \mathrm{mg}, 94.0 \mu \mathrm{mol}),{ }^{n} \mathrm{Bu}_{3} \mathrm{SnH}(55.7 \mu \mathrm{L}, 0.207 \mathrm{mmol})$ and AIBN $(12.3 \mathrm{mg}, 75.0 \mu \mathrm{mol})$ furnished after workup and chromatography $\left(\mathrm{SiO}_{2}, 4 \%\right.$ ethyl acetate in hexanes), $(\boldsymbol{S})-4$ as a colorless oil $\left(26.0 \mathrm{mg}, 75 \%\right.$ yield). $\mathrm{R}_{f}=0.35$ (10\% EtOAc/hexanes); IR (film) 3055, $1744 \mathrm{~cm}^{-1} ;{ }^{1} \mathrm{H}$ NMR $\left(400 \mathrm{MHz}, \mathrm{CDCl}_{3}\right) \delta 7.47(\mathrm{~d}, J=1.6 \mathrm{~Hz}, 2 \mathrm{H}), 7.46-7.24(\mathrm{~m}$, $8 \mathrm{H}), 7.05(\mathrm{~d}, J=7.2 \mathrm{~Hz}, 1 \mathrm{H}), 6.91(\mathrm{t}, J=7.6 \mathrm{~Hz}, 1 \mathrm{H}), 6.61(\mathrm{t}, J=7.2 \mathrm{~Hz}, 1 \mathrm{H}), 6.07(\mathrm{~d}, J=$ $4.0 \mathrm{~Hz}, 1 \mathrm{H}), 5.83-5.73(\mathrm{~m}, 1 \mathrm{H}), 5.66(\mathrm{~s}, 1 \mathrm{H}), 5.21(\mathrm{~d}, J=1.2 \mathrm{~Hz}, 1 \mathrm{H}), 5.18(\mathrm{dd}, J=4.8,1.2$ $\mathrm{Hz}, 1 \mathrm{H}), 4.44-4.35(\mathrm{~m}, 2 \mathrm{H}), 4.27(\mathrm{dd}, J=10.0,5.6 \mathrm{~Hz}, 1 \mathrm{H}), 3.45(\mathrm{dd}, J=15.6,10.4 \mathrm{~Hz}$, 
1H), $3.07(\mathrm{dd}, J=16.0,5.6 \mathrm{~Hz}, 1 \mathrm{H}) ;{ }^{13} \mathrm{C} \mathrm{NMR}\left(100 \mathrm{MHz}, \mathrm{CDCl}_{3}\right) \mathrm{ppm} \mathrm{173.2,} \mathrm{151.1,} \mathrm{140.8,}$ 140.6, 131.7, 129.0, 128.6, 128.5 (3C), 127.5 (2C), 127.4, 123.9, 118.4, 118.2, 109.3, 67.2, 65.3, 64.5, 34.0; HRMS (EI): Exact mass calcd for $\mathrm{C}_{25} \mathrm{H}_{23} \mathrm{NO}_{2}[\mathrm{M}]^{+}$369.1729. Found 369.1745. HPLC (Chiralcel AD, 2\% ${ }^{i} \operatorname{PrOH} /$ hexanes, $\left.1 \mathrm{mLmin}\right): \mathrm{t}_{\mathrm{r}}(R)=6.6 \mathrm{~m}, \mathrm{t}_{\mathrm{r}}(S)=10.6 \mathrm{~m} .(S)-$ $38 \%$ ee $[\alpha]_{\mathrm{D}}^{21}-17.9^{\circ}\left(c 0.7, \mathrm{CHCl}_{3}\right)$.
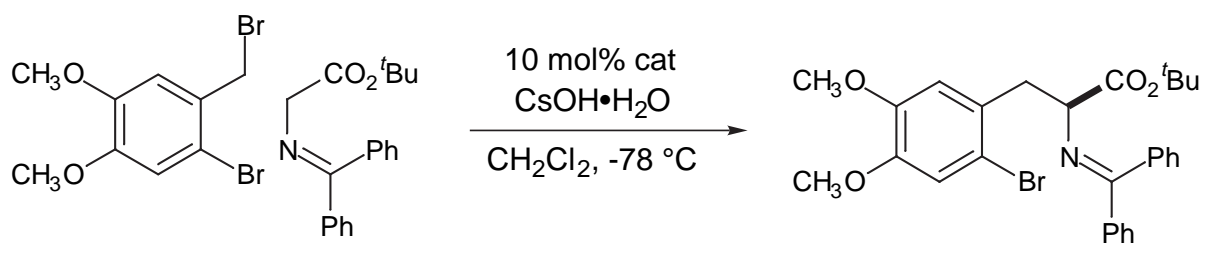

(S)-2-(Benzhydrylideneamino)-3-(2-bromo-4,5-dimethoxyphenyl)propionic acid tert-butyl ester (5i). Following general procedure, 1-bromo-2-bromomethyl-4,5dimethoxybenzene $^{10}(787 \mathrm{mg}, 2.54 \mathrm{mmol})$, the glycinyl imine $(150 \mathrm{mg}, 0.508 \mathrm{mmol})$, and $\mathrm{CsOH} \cdot \mathrm{H}_{2} \mathrm{O}(757 \mathrm{mg}, 5.08 \mathrm{mmol})$ were stirred in $\mathrm{CH}_{2} \mathrm{Cl}_{2}$ for $15 \mathrm{~h}$ in the presence of the cinchonidine derived catalyst $(10 \mathrm{~mol} \%)$ while maintaining a temperature $\leq-65{ }^{\circ} \mathrm{C}$. Work-up according to the general procedure provided the desired product as a white solid (226 $\mathrm{mg}, 85 \%$ ). mp 99-100 ${ }^{\circ} \mathrm{C} ; \mathrm{R}_{f}=0.13$ (10\% EtOAc/hexanes); IR (film) 3057, $1732 \mathrm{~cm}^{-1} ;{ }^{1} \mathrm{H}$ NMR (400 MHz, $\left.\mathrm{CDCl}_{3}\right) \delta 7.63(\mathrm{~d}, J=7.3 \mathrm{~Hz}, 2 \mathrm{H}), 7.39-7.28(\mathrm{~m}, 6 \mathrm{H}), 6.92(\mathrm{~s}, 1 \mathrm{H}), 6.71(\mathrm{~s}, 1 \mathrm{H}), 6.62$ (br d, $J$ $=6.5 \mathrm{~Hz}, 2 \mathrm{H}), 4.29(\mathrm{dd}, J=9.9,3.8 \mathrm{~Hz}, 1 \mathrm{H}), 3.85(\mathrm{~s}, 3 \mathrm{H}), 3.63(\mathrm{~s}, 3 \mathrm{H}), 3.39(\mathrm{dd}, J=13.4$, $3.8 \mathrm{~Hz}, 1 \mathrm{H}), 3.20(\mathrm{dd}, J=13.3,10.1 \mathrm{~Hz}, 1 \mathrm{H}), 1.48$ (s 9H); ${ }^{13} \mathrm{C} \mathrm{NMR}\left(100 \mathrm{MHz}, \mathrm{CDCl}_{3}\right) \mathrm{ppm}$ $170.6,170.4,148.0,147.7,139.3,136.0,130.2,129.5,128.7,128.2,128.0,127.9,127.7$, 115.0, 114.8, 81.2, 65.4, 56.1, 55.7, 39.0, 28.0; HRMS (EI): Exact mass calcd for $\mathrm{C}_{28} \mathrm{H}_{31} \mathrm{BrNO}_{4}[\mathrm{M}+\mathrm{H}]^{+}$, 524.1436. Found 524.1418.

Similarly, use of cinchonine derived catalyst gave the enantiomer as a white solid (256 mg, $95 \%)$

HPLC (Chiralcel OD, 2\% ${ }^{i} \operatorname{PrOH} /$ hexanes, $\left.1 \mathrm{mLmin}\right): \mathrm{t}_{\mathrm{r}}(S)=7.1 \mathrm{~m}, \mathrm{t}_{\mathrm{r}}(R)=8.5 \mathrm{~m} .(S)-$ $92 \%$ ee, $[\alpha]_{\mathrm{D}}^{23}-202.5^{\circ}\left(c 1.4, \mathrm{CHCl}_{3}\right) .(R)-97 \%$ ee, $[\alpha]_{\mathrm{D}}^{25}+214.3^{\circ}\left(c 0.8, \mathrm{CHCl}_{3}\right)$.

\footnotetext{
${ }^{10}$ Landais, Y.; Robin, J. P.; Lebron, A. Tetrahedron 1991, 47, 3787.
} 

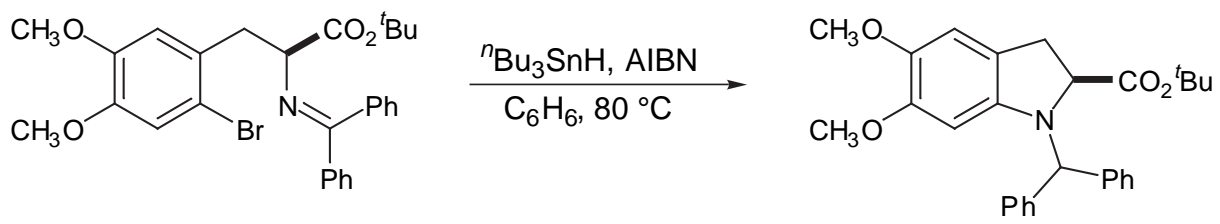

(S)-1-Benzhydryl-5,6-dimethoxy-2,3-dihydro-1H-indole-2-carboxylic acid tertbutyl ester (6i). Following the general procedure, the ketimine $(41.6 \mathrm{mg}, 79.0 \mu \mathrm{mol}),{ }^{\mathrm{n}} \mathrm{Bu}_{3} \mathrm{SnH}$ (107 $\mu \mathrm{L}, 397 \mu \mathrm{mol})$, and AIBN (15.6 mg, $95.0 \mu \mathrm{mol})$ gave, following chromatography (neutral alumina, $5 \%$ EtOAc in hexanes), the indoline as a colorless oil $(18.0 \mathrm{mg}, 50 \%) . \mathrm{R}_{f}=0.13(10 \%$ EtOAc/hexanes); IR (film) 3061, $1733 \mathrm{~cm}^{-1} ;{ }^{1} \mathrm{H}$ NMR (400 MHz, $\left.\mathrm{CDCl}_{3}\right) \delta 7.45$ (dd, $J=6.9$, $5.5 \mathrm{~Hz}, 4 \mathrm{H}), 7.34-7.22(\mathrm{~m}, 6 \mathrm{H}), 6.67(\mathrm{~s}, 1 \mathrm{H}), 5.65(\mathrm{~s}, 1 \mathrm{H}), 5.62$ (s 1H), 4.10 (dd, $J=10.1$, $5.2 \mathrm{~Hz}, 1 \mathrm{H}), 3.78(\mathrm{~s}, 3 \mathrm{H}), 3.41(\mathrm{~s}, 3 \mathrm{H}), 3.35(\mathrm{dd}, J=15.6,10.3 \mathrm{~Hz}, 1 \mathrm{H}), 2.93$ (dd, $J=15.0$, $5.1 \mathrm{~Hz}, 1 \mathrm{H}), 1.36(\mathrm{~s}, 9 \mathrm{H}) ;{ }^{13} \mathrm{C} \mathrm{NMR}\left(100 \mathrm{MHz}, \mathrm{CDCl}_{3}\right) \mathrm{ppm}$ 172.6, 148.4, 145.6, 141.7, $141.5,140.7,132.4,130.1129 .1,128.5,128.4,128.3,127.4,127.2,118.2,109.8,96.3$, 80.9, 67.5, 65.5, 57.0, 55.5, 33.7, 27.9; HRMS (EI): Exact mass calcd for $\mathrm{C}_{28} \mathrm{H}_{31} \mathrm{NO}_{4}[\mathrm{M}]^{+}$, 445.2253. Found, 445.2167.

The enantiomeric indoline was similarly prepared $(27.7 \mathrm{mg}, 50 \%)$.

HPLC (Chiralcel AD, 2\% ${ }^{i} \operatorname{PrOH} /$ hexanes, $\left.1 \mathrm{mLmin}\right): \mathrm{t}_{\mathrm{r}}(R)=7.9 \mathrm{~m}, \mathrm{t}_{\mathrm{r}}(S)=8.6 \mathrm{~m} .(S)-$ $82 \%$ ee, $[\alpha]_{\mathrm{D}}^{25}-18.0^{\circ}\left(c 0.8, \mathrm{CHCl}_{3}\right) .(R)-82 \%$ ee, $[\alpha]_{\mathrm{D}}^{25}+18.5^{\circ}\left(c 2.2, \mathrm{CHCl}_{3}\right)$.

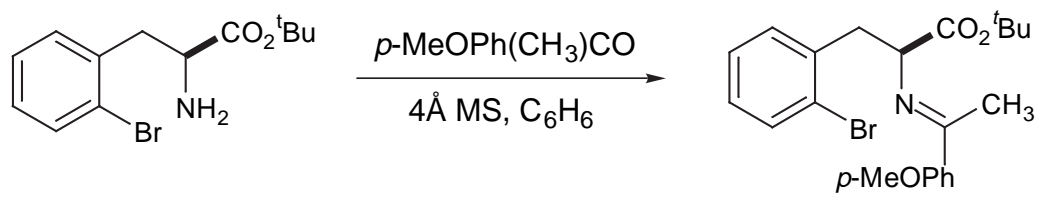

(S)-3-(2-bromophenyl)-2-[1-(4-methoxyphenyl)-ethylideneamino]-propionic acid tert-butyl ester (5j). Following the general procedure, amine S-1 (107 mg, $356 \mu \mathrm{mol}$ ), paramethoxyacetophenone $(53.0 \mathrm{mg}, 356 \mu \mathrm{mol})$, and $4 \AA \mathrm{MS}$ in toluene $(0.36 \mathrm{~mL})$ after $36 \mathrm{~h}$ at reflux provided the desired ketimine as a colorless oil (115 mg, 75\%) and > 95:5 mixture of stereoisomers ( ${ }^{1} \mathrm{H}$ NMR); IR (film) 2974, 1730, $1678 \mathrm{~cm}^{-1} ;{ }^{1} \mathrm{H}$ NMR (400 MHz, $\left.\mathrm{CDCl}_{3}\right) \delta 7.70$ $(\mathrm{d}, J=8.9 \mathrm{~Hz}, 2 \mathrm{H}), 7.28-7.21(\mathrm{~m}, 2 \mathrm{H}), 7.17-7.11(\mathrm{~m}, 2 \mathrm{H}), 6.85(\mathrm{~d}, J=8.9 \mathrm{~Hz}, 2 \mathrm{H}), 4.58(\mathrm{dd}$, 
$J=8.9,5.5 \mathrm{~Hz}, 1 \mathrm{H}), 3.85(\mathrm{~s}, 3 \mathrm{H}), 3.55(\mathrm{dd}, J=13.4,5.2 \mathrm{~Hz}, 1 \mathrm{H}), 3.15(\mathrm{dd}, J=13.4,8.9 \mathrm{~Hz}$, 1H), $1.91(\mathrm{~s}, 3 \mathrm{H}), 1.42(\mathrm{~s}, 9 \mathrm{H}) ;{ }^{13} \mathrm{C}$ NMR (100 MHz, $\left.\mathrm{CDCl}_{3}\right) \mathrm{ppm} 166.8,137.7,132.8,130.1$, $129.0,128.5,128.3,128.2,128.1,127.3,113.6,113.3,81.1,63.8,55.3,40.0,29.7,28.0$; HRMS (EI): Exact mass calcd for $\mathrm{C}_{22} \mathrm{H}_{27} \mathrm{BrNO}_{3}[\mathrm{M}+\mathrm{H}]^{+}$, 432.1174. Found 432.1172. $(S)-87 \%$ ee $[\alpha]_{\mathrm{D}}^{21}-43.1^{\circ}\left(c 0.7, \mathrm{CHCl}_{3}\right)$.

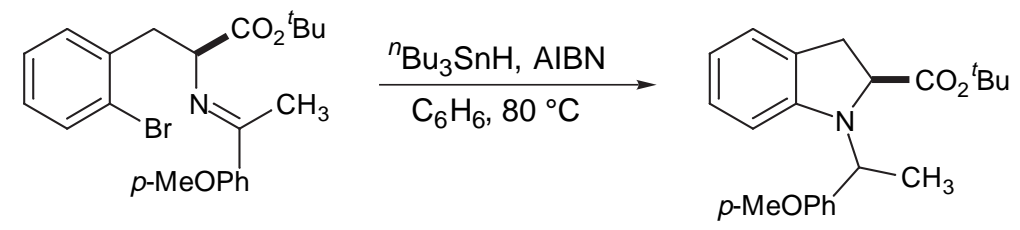

(S)-1-[1-(4-methoxy phenyl) ethyl]-2,3-dihydro-1H-indole-2-carboxylic acid tertbutyl ester (6j). Following the general procedure, the ketimine $(36.0 \mathrm{mg}, 86.0 \mu \mathrm{mol}),{ }^{\mathrm{n}} \mathrm{Bu}_{3} \mathrm{SnH}$ $(51.0 \mu \mathrm{L}, 189 \mu \mathrm{mol})$, and AIBN $(11.3 \mathrm{mg}, 69.0 \mu \mathrm{mol})$ furnished, after silica gel chromatography (1\% ether in hexanes), the desired indoline $(15.0 \mathrm{mg}, 50 \%)$ as a 3:1 mixture of diastereomers.

Minor diastereomer: $\mathrm{R}_{f}=0.55$ (10\% EtOAc/hexanes); IR (film) 2974, $1741 \mathrm{~cm}^{-1} ;{ }^{1} \mathrm{H}$ NMR (400 $\left.\mathrm{MHz}, \mathrm{CDCl}_{3}\right) \delta 7.45(\mathrm{~d}, J=8.6 \mathrm{~Hz}, 2 \mathrm{H}), 7.01(\mathrm{~d}, J=7.0 \mathrm{~Hz}, 1 \mathrm{H}), 6.95(\mathrm{t}, \mathrm{J}=7.6 \mathrm{~Hz}, 1 \mathrm{H})$, $6.86(\mathrm{~d}, J=8.6 \mathrm{~Hz}, 2 \mathrm{H}), 6.63(\mathrm{t}, J=7.0 \mathrm{~Hz}, 1 \mathrm{H}), 6.26(\mathrm{~d}, J=7.9 \mathrm{~Hz}, 1 \mathrm{H}), 4.49(\mathrm{q}, J=7.0$ $\mathrm{Hz}, 1 \mathrm{H}), 4.36(\mathrm{t}, J=9.5 \mathrm{~Hz}, 1 \mathrm{H}), 3.80(\mathrm{~s}, 3 \mathrm{H}), 3.42(\mathrm{dd}, J=15.9,10.4 \mathrm{~Hz}, 1 \mathrm{H}), 3.06(\mathrm{dd}, J=$ 15.9, 9.2 Hz, 1H), $1.51(\mathrm{~d}, J=7.0 \mathrm{~Hz}, 3 \mathrm{H}), 1.39(\mathrm{~s}, 9 \mathrm{H}) ;{ }^{13} \mathrm{C} \mathrm{NMR}\left(100 \mathrm{MHz}, \mathrm{CDCl}_{3}\right) \mathrm{ppm}$ $173.5,158.5,151.7,136.2,128.2(2 \mathrm{C}), 127.5,123.8,117.9,113.8,108.6,81.0,64.2,57.6$, 55.2, 34.5, 27.9, 19.3; HRMS (EI): Exact mass calcd for $\mathrm{C}_{22} \mathrm{H}_{27} \mathrm{NO}_{3}[\mathrm{M}]^{+}, 353.1991$. Found 353.2008 .

HPLC (Chiralcel OD, 2\% ${ }^{i} \mathrm{PrOH} /$ hexanes, $\left.0.5 \mathrm{mLmin}\right): \mathrm{t}_{\mathrm{r}}(2 S)=11.0 \mathrm{~m}, \mathrm{t}_{\mathrm{r}}(2 R)=13.2 \mathrm{~m}$. $(S)-32 \%$ ee, $[\alpha]_{\mathrm{D}}^{21}+2.1^{\circ}\left(c 0.5, \mathrm{CHCl}_{3}\right)$.

Major diastereomer: $\mathrm{R}_{f}=0.45\left(10 \%\right.$ EtOAc/hexanes); ${ }^{1} \mathrm{H} \mathrm{NMR}\left(400 \mathrm{MHz}, \mathrm{CDCl}_{3}\right) \delta 7.41(\mathrm{~d}, J=$ $8.7 \mathrm{~Hz}, 2 \mathrm{H}), 6.99(\mathrm{~d}, J=7.0 \mathrm{~Hz}, 1 \mathrm{H}), 6.87-6.83(\mathrm{~m}, 3 \mathrm{H}), 6.58(\mathrm{t}, J=6.9 \mathrm{~Hz}, 1 \mathrm{H}), 6.08(\mathrm{~d}, J$ $=7.9 \mathrm{~Hz}, 1 \mathrm{H}), 4.60(\mathrm{q}, J=7.0 \mathrm{~Hz}, 1 \mathrm{H}), 4.28(\mathrm{dd}, J=10.3,7.4 \mathrm{~Hz}, 1 \mathrm{H}), 3.80(\mathrm{~s}, 3 \mathrm{H}), 3.37$ $(\mathrm{dd}, J=16.0,10.5 \mathrm{~Hz}, 1 \mathrm{H}), 3.08(\mathrm{dd}, J=15.9,7.4 \mathrm{~Hz}, 1 \mathrm{H}), 1.51(\mathrm{~d}, J=6.9 \mathrm{~Hz}, 3 \mathrm{H}), 1.47(\mathrm{~s}$, 
9H) $;{ }^{13} \mathrm{C} \mathrm{NMR}\left(100 \mathrm{MHz}, \mathrm{CDCl}_{3}\right) \mathrm{ppm} 173.4,148.1,139.3,127.9$ (2C), 127.2, 124.0, 117.5, 113.7 (2C), 108.9, 64.4, 62.9, 55.2, 55.1, 33.8, 28.0, 16.5 .

Anal. Calcd for $\mathrm{C}_{22} \mathrm{H}_{27} \mathrm{NO}_{3}$ : C, 74.76; H, 7.70; N, 3.96. Found: C, 74.77; H, 7.77; N, 3.95 .

HPLC (Chiralcel OD, 2\% $\operatorname{PrOH} /$ hexanes, $\left.^{\circ} 0.5 \mathrm{mLmin}\right): \mathrm{t}_{\mathrm{r}}(2 S)=13.0 \mathrm{~m}, \mathrm{t}_{\mathrm{r}}(2 R)=15.0 \mathrm{~m}$. $(S)-32 \%$ ee, $[\alpha]_{\mathrm{D}}^{21}-7.2^{\circ}\left(c 1.1, \mathrm{CHCl}_{3}\right)$.

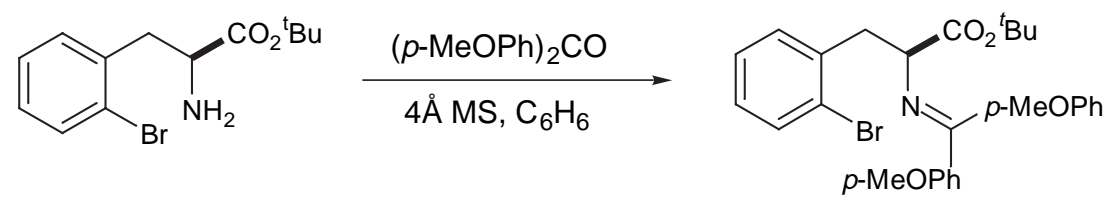

2-\{[bis-(4-Methoxyphenyl)-methylene]-amino-3-(2-bromophenyl)-propionic acid tert-butyl ester (5k). According to the general procedure, use of S-1 $(52.2 \mathrm{mg}, 175 \mu \mathrm{mol})$, bis-4-methoxybenzophenone imine (41.6 mg, $173 \mu \mathrm{mol}), 4 \AA$ sieves $(1.5 \mathrm{~g})$, and benzene $(6 \mathrm{ml})$, gave the desired product as a white solid $(87 \mathrm{mg}, 96 \%)$. mp 109-113 ${ }^{\circ} \mathrm{C}$; IR (film) $2975,1730 \mathrm{~cm}^{-}$ ${ }^{1} ;{ }^{1} \mathrm{H}$ NMR $\left(400 \mathrm{MHz}, \mathrm{CDCl}_{3}\right) \delta 7.53(\mathrm{~d}, J=8.6 \mathrm{~Hz}, 2 \mathrm{H}), 7.42(\mathrm{~d}, J=7.8 \mathrm{~Hz}, 1 \mathrm{H}), 7.19(\mathrm{~d}, J=$ $6.7 \mathrm{~Hz}, 1 \mathrm{H}), 7.11(\mathrm{t}, J=7.3 \mathrm{~Hz}, 1 \mathrm{H}), 7.01(\mathrm{t}, J=7.0 \mathrm{~Hz}, 1 \mathrm{H}), 6.80(\mathrm{~m}, 2 \mathrm{H}), 6.53(\mathrm{~d}, J=7$ $\mathrm{Hz}, 2 \mathrm{H}), 4.35$ (dd, $J=9.7,4.0 \mathrm{~Hz}, 1 \mathrm{H}), 3.83$ (s, 3H), 3.80 (s, 3H), $3.43(\mathrm{dd}, J=13.4,4.0 \mathrm{~Hz}$, 1H), $3.20(\mathrm{~m}, 1 \mathrm{H}), 1.45$ (s, 9H); ${ }^{13} \mathrm{C}$ NMR (100 MHz) ppm 171.2, 161.4, 159.6, 138.0, 134.2, $132.9,132.7,130.7,129.4,128.8,128.1,127.1,125.4,114.9,113.6,113.4,81.3,65.2$, 55.5, 39.9, 28.3; HRMS (CI, $\mathrm{CH}_{4}$ ) Exact mass calcd for $\mathrm{C}_{28} \mathrm{H}_{31}{ }^{81} \mathrm{BrNO}_{4}[\mathrm{M}+\mathrm{H}]^{+}$524.1438. Found 526.1419.
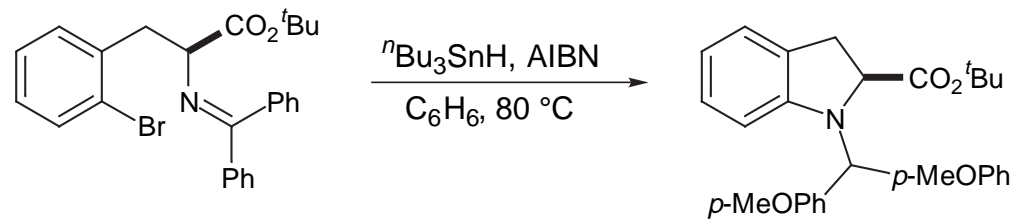

1-[bis-(4-methoxy-phenyl)-methyl]-2,3-dihydro-1H-indole-2-carboxylic acid tertbutyl ester (6k). According to the general procedure, use of 5k $(31.1 \mathrm{mg}, 59.5 \mu \mathrm{mol})$, 
${ }^{n} \mathrm{Bu}_{3} \mathrm{SnH}(35 \mu \mathrm{L}, 330 \mu \mathrm{mol})$, and AIBN $(7.8 \mathrm{mg}, 47.6 \mu \mathrm{mol})$ provided the desired indoline after flash chromatography $\left(\mathrm{SiO}_{2}, 2 \%\right.$ ethyl acetates in hexanes) as a colorless oil (18.7 $\left.\mathrm{mg}, 71 \%\right)$. $\mathrm{R}_{f}=0.18$ (10\% ethyl acetate in hexanes); IR (film) 2929, 1735, $1510 \mathrm{~cm}^{-1}$; ${ }^{1} \mathrm{H}$ NMR (400 MHz, $\left.\mathrm{CDCl}_{3}\right) \delta 7.36(\mathrm{~d}, J=8.7 \mathrm{~Hz}, 2 \mathrm{H}), 7.23(\mathrm{~d}, J=8.6 \mathrm{~Hz}, 2 \mathrm{H}), 7.00(\mathrm{~d}, J=6.6 \mathrm{~Hz}, 1 \mathrm{H}), 6.83(\mathrm{~m}$, $5 \mathrm{H}), 6.61(\mathrm{dd}, J=13.9,7.0 \mathrm{~Hz}, 1 \mathrm{H}), 6.01(\mathrm{~d}, J=7.8 \mathrm{~Hz}, 1 \mathrm{H}), 5.54(\mathrm{~s}, 1 \mathrm{H}), 4.08(\mathrm{dd}, J=$ $10.5,5.9 \mathrm{~Hz}, 1 \mathrm{H}), 3.79(\mathrm{~s}, 3 \mathrm{H}), 3.78(\mathrm{~s}, 3 \mathrm{H}), 3.38(\mathrm{dd}, J=15.8,10.5 \mathrm{~Hz}, 1 \mathrm{H}), 2.98(\mathrm{dd}, J=$ 15.8, $5.8 \mathrm{~Hz}, 1 \mathrm{H}), 1.34$ (s, 9H); ${ }^{13} \mathrm{C}$ NMR (100 MHz) ppm 173.0, 158.9, 151.5, 133.8, 133.6, $130.3,129.8,128.0,127.6,124.0,118.0,114.0,109.5,81.1,66.1,65.2,55.5,34.3,30.0$, 28.2; HRMS (EI): Exact mass calcd for $\mathrm{C}_{28} \mathrm{H}_{31} \mathrm{NO}_{4}[\mathrm{M}]^{+}$, 445.2253. Found 445.2263.

Anal. Calcd for $\mathrm{C}_{28} \mathrm{H}_{31} \mathrm{NO}_{4}$ : C, 75.48; H, 7.11; N, 3.17. Found: C, 75.19; H, 7.11; N, 3.17 .

HPLC: Chiralcel AD (2\% ${ }^{i} \mathrm{PrOH} /$ hexanes, $\left.1 \mathrm{~mL} / \mathrm{min}, 254 \mathrm{~nm}\right), \mathrm{t}_{\mathrm{r}}\left(\right.$ Peak 1) $12.9 \mathrm{~min}, \mathrm{t}_{\mathrm{r}}$ (Peak 2) $17.8 \mathrm{~min} ; 83.9 \%$ ee: $[\alpha]_{\mathrm{D}}^{21}-12.2^{\circ}\left(c 0.8, \mathrm{CDCl}_{3}\right)$.

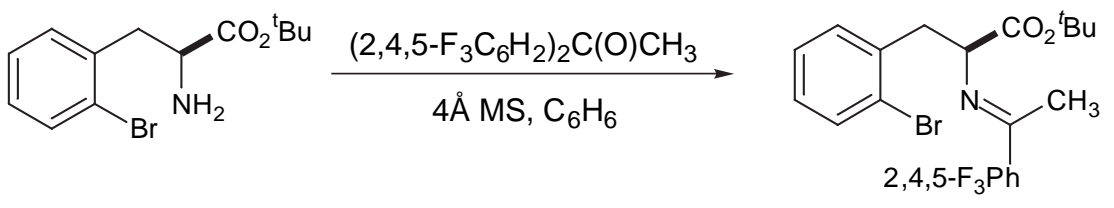

(S)-3-(2-Bromophenyl)-2-[1-(2',4',5'-trifluorophenyl)-ethylideneamino]-

propionic acid tert-butyl ester (5l). Following the general procedure, amine S-1 (107 mg, $356 \mu \mathrm{mol}), 2^{\prime} 4^{\prime} 5^{\prime}$-trifluoroacetophenone $(46.6 \mu \mathrm{L}, 356 \mu \mathrm{mol})$, and $4 \AA \mathrm{MS}$ in toluene $(0.36 \mathrm{~mL})$ after $30 \mathrm{~h}$ at reflux provided the desire ketimine (115 mg, 70\%) as a colorless oil; IR (film) 3065 , 1734, $1618 \mathrm{~cm}^{-1} ;{ }^{1} \mathrm{H}$ NMR (400 MHz, $\left.\mathrm{CDCl}_{3}\right) \delta 7.56(\mathrm{~d}, J=7.7 \mathrm{~Hz}, 1 \mathrm{H}), 7.24-7.23(\mathrm{~m}, 2 \mathrm{H})$, 7.14-7.10 (m, 2H), $6.90(\mathrm{td}, J=9.9,6.3 \mathrm{~Hz}, 1 \mathrm{H}), 4.65(\mathrm{dd}, J=9.1,5.1 \mathrm{~Hz}, 1 \mathrm{H}), 3.61(\mathrm{dd}, J=$ 13.4, 5.1Hz, 1H), $3.19(\mathrm{dd}, J=13.4,9.1 \mathrm{~Hz}, 1 \mathrm{H}), 1.96(\mathrm{~d}, J=3.4 \mathrm{~Hz}, 3 \mathrm{H}), 1.48(\mathrm{~s}, 9 \mathrm{H}) ;{ }^{13} \mathrm{C}$ NMR (100 MHz, $\mathrm{CDCl}_{3}$ ) ppm 169.9, 165.0, 155.2, 150.6, 137.1, 132.6 (2C), 128.4, 127.1, 127.0, 124.7, $117.8\left(J_{\mathrm{C}-\mathrm{F}}=14.5 \mathrm{~Hz}, 1 \mathrm{C}\right), 105.7(\mathrm{~m}, 2 \mathrm{C}), 81.7,65.6,63.3,39.6,28.0$; HRMS (EI): Exact mass calcd for $\mathrm{C}_{21} \mathrm{H}_{22} \mathrm{BrF}_{3} \mathrm{NO}_{2}[\mathrm{M}+\mathrm{H}]^{+}$, 456.0786. Found 456.0781. 
HPLC (Chiralcel OD, 2\% ${ }^{i} \operatorname{PrOH} /$ hexanes, $\left.0.5 \mathrm{~mL} / \mathrm{min}\right): \mathrm{t}_{\mathrm{r}}(S)=8.6 \mathrm{~m}, \mathrm{t}_{\mathrm{r}}(R)=11.8 \mathrm{~m}$. $(S)-87 \%$ ee, $[\alpha]_{\mathrm{D}}^{21}-66.4^{\circ}\left(c 0.6, \mathrm{CHCl}_{3}\right)$.

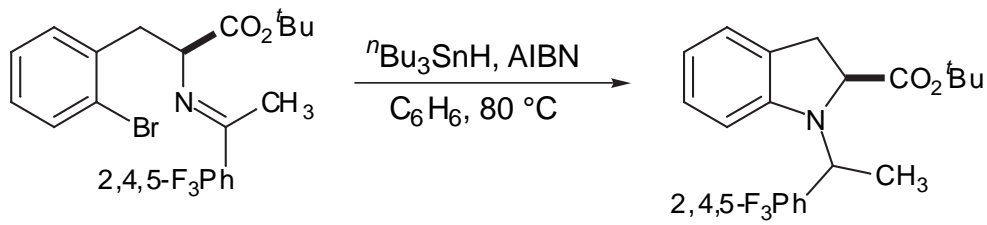

(S)-1-[1-(2,4,5-trifluorophenyl)ethyl]-2,3-dihydro-1H-indole-2-carboxylic acid tert-butyl ester (61). Following the general procedure, the ketimine (90.0 $\mathrm{mg}, 196 \mu \mathrm{mol})$, ${ }^{n} \mathrm{Bu}_{3} \mathrm{SnH}(116 \mu \mathrm{L}, 434 \mu \mathrm{mol})$, and AIBN (26.0 mg, $\left.158 \mu \mathrm{mol}\right)$ furnished after silica gel chromatography (1\% ethyl acetate in hexanes) the desired indoline $(52.0 \mathrm{mg}, 70 \%)$ as a colorless oil and a 3:1 inseparable mixture of diastereomers. $R_{f}=0.58$ (10\% EtOAc/hexanes); IR (film) 3062, $1737 \mathrm{~cm}^{-1} ;{ }^{1} \mathrm{H}$ NMR (400 MHz, $\left.\mathrm{CDCl}_{3}\right) \delta$ 7.87-7.80 (m, 1H), $7.05(\mathrm{~d}, J=7.3 \mathrm{~Hz}, 1 \mathrm{H})$, $7.00-6.91(\mathrm{~m}, 2 \mathrm{H}), 6.70(\mathrm{t}, J=6.8 \mathrm{~Hz}, 1 \mathrm{H}), 6.08(\mathrm{~d}, J=7.9 \mathrm{~Hz}, 1 \mathrm{H}), 4.71(\mathrm{dd}, J=14.0,7.0$ $\mathrm{Hz}, 1 \mathrm{H}), 4.48(\mathrm{t}, J=10.2 \mathrm{~Hz}, 1 \mathrm{H}), 3.48(\mathrm{dd}, J=15.7,10.2 \mathrm{~Hz}, 1 \mathrm{H}), 3.13(\mathrm{dd}, J=15.8,10.1$ $\mathrm{Hz}, 1 \mathrm{H}), 1.51-1.50(\mathrm{~m}, 12 \mathrm{H}) ;{ }^{13} \mathrm{C} \mathrm{NMR}\left(100 \mathrm{MHz}, \mathrm{CDCl}_{3}\right) \mathrm{ppm} 173.4,151.1,128.5,127.7$, $127.4,127.0,124.0,123.9,118.6,118.1\left(\mathrm{~d} J_{\mathrm{C}-\mathrm{F}}=19.7 \mathrm{~Hz}, 1 \mathrm{C}\right), 107.9,105.3$ (m 2C), 81.7, 64.1, 51.9, 34.5, 27.9, 18.4; HRMS (EI): Exact mass calcd for $\mathrm{C}_{21} \mathrm{H}_{22} \mathrm{NF}_{3} \mathrm{O}_{2}[\mathrm{M}]^{+}, 377.1603$. Found 377.1599.

Anal. Calcd for $\mathrm{C}_{21} \mathrm{H}_{22} \mathrm{NF}_{3} \mathrm{O}_{2}: \mathrm{C}, 66.83 ; \mathrm{H}, 5.88 ; \mathrm{N}, 3.71$. Found: C, 66.86; H, 5.80; N, 3.62 .

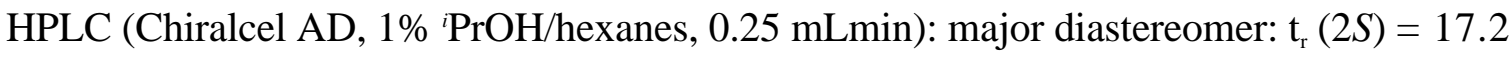
$\mathrm{m}, \mathrm{t}_{r}(2 R)=20.2 \mathrm{~m}$; minor diastereomer $: \mathrm{t}_{\mathrm{r}}(2 S)=18.6 \mathrm{~m}, \mathrm{t}_{\mathrm{r}}(2 R)=19.5 \mathrm{~m} .(2 S)-82 \%$ ee, $[\alpha]_{\mathrm{D}}^{21}$ $-4.9^{\circ}\left(c 0.7, \mathrm{CHCl}_{3}\right)$. 


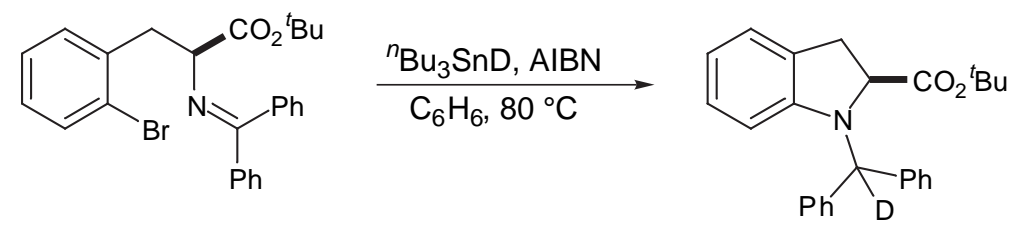

(S)-1-Benzhydryl-2,3-dihydro-1D-indole-2-carboxylic acid tert-butyl ester (9).

According to the general procedure, the Schiff base (18.6 mg, $40.0 \mu \mathrm{mol}),{ }^{n} \mathrm{Bu}_{3} \mathrm{SnH}(23.8 \mu \mathrm{L}$, $0.088 \mathrm{mmol})$ and $\operatorname{AIBN}(5.25 \mathrm{mg}, 0.032 \mathrm{mmol})$ furnished after chromatography $\left(\mathrm{SiO}_{2}, 2 \%\right.$ ethyl acetate in hexanes) the desired product as a colorless oil $(13.9 \mathrm{mg}, 90 \%$ yield $) . \mathrm{R}_{f}=0.42(10 \%$ EtOAc/hexanes); IR (film) 3061, $1730 \mathrm{~cm}^{-1} ;{ }^{1} \mathrm{H}$ NMR (400 MHz, $\left.\mathrm{CDCl}_{3}\right) \delta 7.47$ (d, $J=7.1 \mathrm{~Hz}$, 2H), $7.37(\mathrm{~d}, J=7.1 \mathrm{~Hz}, 2 \mathrm{H}), 7.34-7.23(\mathrm{~m}, 6 \mathrm{H}), 7.03(\mathrm{~d}, J=7.1 \mathrm{~Hz}, 1 \mathrm{H}), 6.87(\mathrm{t}, J=7.7$ $\mathrm{Hz}, 1 \mathrm{H}), 6.64(\mathrm{t}, J=7.3 \mathrm{~Hz}, 1 \mathrm{H}), 6.01(\mathrm{~d}, J=7.9 \mathrm{~Hz}, 1 \mathrm{H}), 4.11(\mathrm{dd} J=10.5,5.4 \mathrm{~Hz}, 1 \mathrm{H})$, $3.42(\mathrm{dd}, J=15.9,10.5 \mathrm{~Hz}, 1 \mathrm{H}), 3.00(\mathrm{dd}, J=15.9,5.4 \mathrm{~Hz}, 1 \mathrm{H}), 1.33(\mathrm{~s}, 9 \mathrm{H}) ;{ }^{2} \mathrm{H}$ NMR $(60$ $\left.\mathrm{MHz}, \mathrm{CDCl}_{3}\right) \delta 5.60(\mathrm{br} \mathrm{s}, 1 \mathrm{H}) ;{ }^{13} \mathrm{C}$ NMR $\left(100 \mathrm{MHz}, \mathrm{CDCl}_{3}\right)$ ppm 172.6, 151.2, 141.1, 140.6, $129.2,128.5$ (2C), $128.4(2 \mathrm{C}), 127.7,127.3,127.2,123.8,117.9,109.2,80.8,66.6(\mathrm{t}, J=$ $21.3 \mathrm{~Hz}$ ), 65.0, 34.0, 27.9; HRMS (EI): Exact mass calcd for $\mathrm{C}_{26} \mathrm{H}_{26} \mathrm{DNO}_{2}[\mathrm{M}]^{+}, 386.2104$. Found 386.2102.
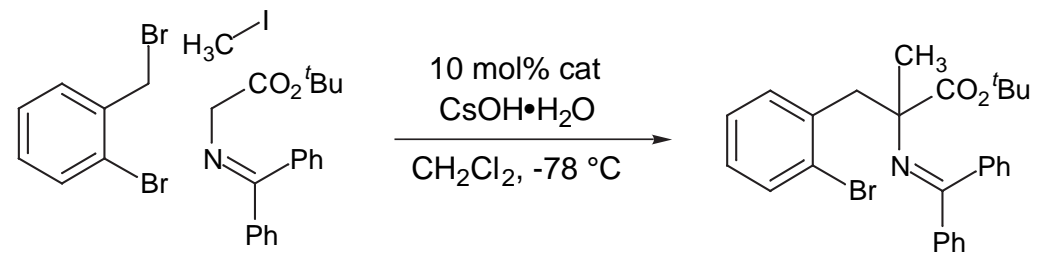

\section{2-(Benzhydrylidene-amino)-3-(2-bromophenyl)-2-methyl-propionic acid tert-butyl} ester (10). As an adaptation of the general procedure, methyl iodide $(22.2 \mu \mathrm{L}, 355 \mu \mathrm{mol})$, Schiff base $(100 \mathrm{mg}, 339 \mu \mathrm{mol}), \mathrm{CsOH} \cdot \mathrm{H}_{2} \mathrm{O}(126 \mathrm{mg}, 845 \mu \mathrm{mol})$, and cinchonidine derived catalyst (20.6 mg, $34.0 \mu \mathrm{mol}$ ), were stirred in toluene $(1 \mathrm{~mL})$ at room temperature for $3.5 \mathrm{~h}$. Additional $\mathrm{CsOH} \cdot \mathrm{H}_{2} \mathrm{O}(252 \mathrm{mg}, 1.69 \mathrm{mmol})$, catalyst $(20.6 \mathrm{mg}, 34.0 \mu \mathrm{mol})$ and ortho-bromobenzyl bromide (114 mg, $407 \mu \mathrm{mol}$ ), were added and the reaction mixture was stirred vigorously for an additional $18 \mathrm{~h}$ at room temperature. The reaction mixture was diluted with ether and water (1:1). The organic 
layer was separated, the aqueous layer was extracted with ether, and the combined ether layers were dried and concentrated. Flash chromatography (neutral alumina, 0.50\% ethyl acetate in hexanes) gave the desired ketimine as a colorless oil $(76.0 \mathrm{mg}, 46 \%)$. The catalyst was recovered by washing the aqueous layer with $\mathrm{CH}_{2} \mathrm{Cl}_{2} . \mathrm{R}_{f}=0.40$ (10\% EtOAc/hexanes); IR (film) 3058, 1730, $1628 \mathrm{~cm}^{-1} ;{ }^{1} \mathrm{H} \mathrm{NMR}\left(400 \mathrm{MHz}, \mathrm{CDCl}_{3}\right) 7.68(\mathrm{dd}, J=7.3,1.6 \mathrm{~Hz}, 1 \mathrm{H}), 7.56(\mathrm{~d}, J=8.4 \mathrm{~Hz}$, 2H), $7.54(\mathrm{t}, J=8.4 \mathrm{~Hz}, 1 \mathrm{H}), 7.34-7.28(\mathrm{~m}, 6 \mathrm{H}), 7.21(\mathrm{td}, J=8.8,0.8 \mathrm{~Hz}, 1 \mathrm{H}), 7.06(\mathrm{~d}, J=$ $6.4 \mathrm{~Hz}, 3 \mathrm{H}), 3.48$ (d, $J=14.0, \mathrm{~Hz}, 1 \mathrm{H}), 3.38$ (d, $J=14.0, \mathrm{~Hz}, 1 \mathrm{H}), 1.32(\mathrm{~s}, 9 \mathrm{H}), 1.17$ (s, 3H); ${ }^{13} \mathrm{C}$ NMR $\left(100 \mathrm{MHz}, \mathrm{CDCl}_{3}\right)$ ppm 173.7, 166.5, 141.5, 138.5, 137.6, 132.6, 129.8, 128.5, 128.5, 128.3, 128.1, 127.9, 127.8 (2C), 126.8, 126.4, 81.0, 68.3, 46.6, 27.9, 23.7; HRMS (EI): Exact mass calcd for $\mathrm{C}_{27} \mathrm{H}_{29} \mathrm{BrNO}_{2}[\mathrm{M}+\mathrm{H}]^{+}$478.1382. Found 478.1363.

HPLC (Chiralcel OD, 1\% ${ }^{i} \mathrm{PrOH} /$ hexanes, $\left.0.5 \mathrm{mLmin}\right): \mathrm{t}_{\mathrm{r}}($ minor $)=8.9 \mathrm{~m}, \mathrm{t}_{\mathrm{r}}$ (major) $=$ $10.8 \mathrm{~m} .(S)-22 \%$ ee.
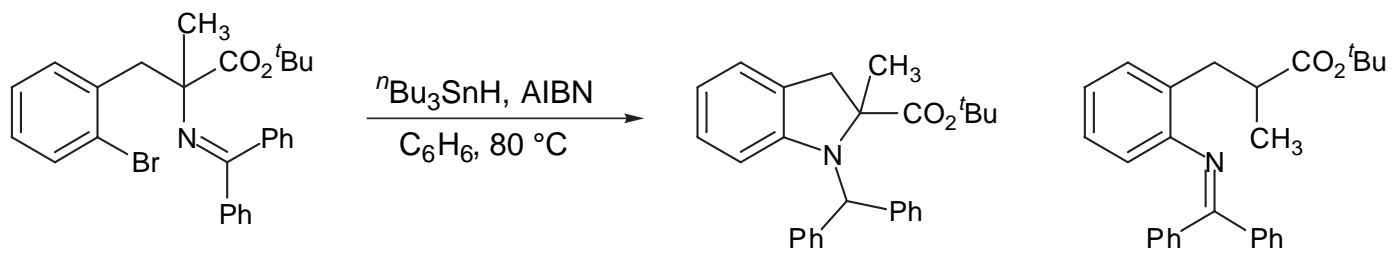

Following the general procedure, Schiff base $(41.0 \mathrm{mg}, 86.0 \mu \mathrm{mol}), n_{\mathrm{Bu}_{3}} \mathrm{SnH}(51.0 \mu \mathrm{L}, 0.189$ mmol) and AIBN (11.3 mg, $69 \mu \mathrm{mol})$ furnished after chromatography (neutral alumina, 20\% dichloromethane in hexanes), the indoline as a colorless oil (16.0 $\mathrm{mg}, 48 \%$ yield) and the aryl imine as a yellow oil (15.0 mg, $45 \%$ yield).

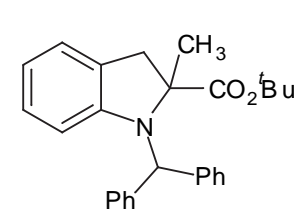

\section{1-Benzhydryl-4-bromo-2-methyl-2,3-dihydro-1H-indole-2-}

carboxylic acid tert-butyl ester (11). $\mathrm{R}_{f}=0.54$ (10\% EtOAc/hexanes); IR (film) 3065, $1727 \mathrm{~cm}^{-1} ;{ }^{1} \mathrm{H}$ NMR (400 MHz, $\left.\mathrm{CDCl}_{3}\right) \delta 7.37-7.26(\mathrm{~m}, 10 \mathrm{H})$, $7.02(\mathrm{~d}, J=7.2 \mathrm{~Hz}, 1 \mathrm{H}), 6.72(\mathrm{t}, J=7.6 \mathrm{~Hz}, 1 \mathrm{H}), 6.54(\mathrm{t}, J=8.0 \mathrm{~Hz}, 1 \mathrm{H})$, $5.80(\mathrm{~s}, 1 \mathrm{H}), 5.75(\mathrm{~d}, J=8.0 \mathrm{~Hz}, 1 \mathrm{H}), 3.67(\mathrm{~d}, J=16.0 \mathrm{~Hz}, 1 \mathrm{H}), 3.03(\mathrm{~d}, J=16.0 \mathrm{~Hz}, 1 \mathrm{H})$, 1.40 (s, 9H), 1.37 (s, 3H); ${ }^{13} \mathrm{C}$ NMR (100 MHz, $\mathrm{CDCl}_{3}$ ) ppm 173.7, 148.7, 142.2, 140.7, $128.64,127.59,128.4,128.1,127.1,126.9,126.8,125.7,123.9,116.2,108.8,81.6,71.2$, 
62.3, 42.5, 27.8, 24.8; HRMS (EI): Exact mass calcd. for $\mathrm{C}_{27} \mathrm{H}_{29} \mathrm{NO}_{2}[\mathrm{M}]^{+}$, 399.2198. Found 399.2185 .

Anal. Calcd for $\mathrm{C}_{27} \mathrm{H}_{29} \mathrm{NO}_{2}: \mathrm{C}, 81.17 ; \mathrm{H}, 7.32 ; \mathrm{N}, 3.51$. Found: C, 81.15; H, 7.52; N, 3.47 .

HPLC (Chiralcel AD, 1\% $\left.{ }^{\text {PrOH} / h e x a n e s, ~} 1.0 \mathrm{mLmin}\right): \mathrm{t}_{\mathrm{r}}=3.8 \mathrm{~m}, 4.0 \mathrm{~m}$.

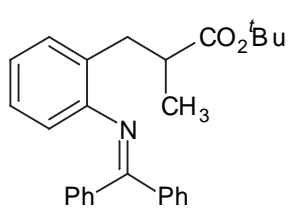

3-[2-(Benzhydrylidene-amino)-phenyl]-2-methyl-propionic acid tert-butyl ester (12). $\mathrm{R}_{f}=0.17 \quad\left(50 \% \quad \mathrm{CH}_{2} \mathrm{Cl}_{2} /\right.$ hexanes); IR (film) 3058 , 1723, $1621 \mathrm{~cm}^{-1} ;{ }^{1} \mathrm{H}$ NMR $\left(400 \mathrm{MHz}, \mathrm{CDCl}_{3}\right) \delta 7.82(\mathrm{~d}, J=7.2 \mathrm{~Hz}, 2 \mathrm{H})$, $7.48(\mathrm{~d}, J=7.2 \mathrm{~Hz}, 1 \mathrm{H}), 7.32-7.25(\mathrm{~m}, 3 \mathrm{H}), 7.15(\mathrm{dd}, J=7.2,1.6 \mathrm{~Hz}, 2 \mathrm{H}), 7.10(\mathrm{~d}, J=7.2$ $\mathrm{Hz}, 1 \mathrm{H}), 6.92(\mathrm{td}, \mathrm{J}=7.2,1.2 \mathrm{~Hz}, 1 \mathrm{H}), 6.87(\mathrm{td}, \mathrm{J}=7.2,1.2 \mathrm{~Hz}, 1 \mathrm{H}), 6.40(\mathrm{~d}, J=8.0 \mathrm{~Hz}$, 1H), 2.95-2.83 (m, 2H), $2.63(\mathrm{dd}, J=12.4,6.8 \mathrm{~Hz}, 1 \mathrm{H}), 1.36(\mathrm{~s}, 9 \mathrm{H}), 1.14(\mathrm{~d}, J=6.8, \mathrm{~Hz}$, 1H); ${ }^{13} \mathrm{C}$ NMR (100 MHz, $\mathrm{CDCl}_{3}$ ) ppm 176.1, 166.6, 139.5, 130.7, 130.5, 129.9, 129.3, 129.0 (2C), 128.6, 128.1, 127.9, 126.3, 123.2, 119.9, 79.8, 39.9, 36.4, 29.7, 28.0, 17.2; HRMS (EI): Exact mass calcd. for $\mathrm{C}_{27} \mathrm{H}_{29} \mathrm{NO}_{2}[\mathrm{M}]^{+}, 399.2198$. Found 399.2212.

Anal. Calcd for $\mathrm{C}_{27} \mathrm{H}_{29} \mathrm{NO}_{2}$ : C, 81.17; H, 7.32; N, 3.51. Found: C, 81.37; H, 7.36; N, 3.46 .

HPLC (Chiralcel AD, 1\% ${ }^{~} \mathrm{PrOH} / \mathrm{h}^{2}$ xanes, $\left.1.0 \mathrm{mLmin}\right): \mathrm{t}_{\mathrm{r}}=4.7 \mathrm{~m}, 5.1 \mathrm{~m}$. 